ESAIM: Proceedings, Vol. 4, 1998, 199-222

Contrôle et Équations aux Dérivées Partielles

http://www.emath.fr/proc/Vol.4/

\title{
ANALYTICITY, AND LACK THEREOF, OF THERMO-ELASTIC SEMIGROUPS ${ }^{1}$
}

\author{
IRENA LASIECKA \\ Applied Mathematics, Thornton Hall \\ University of Virginia \\ Charlottesville, VA 22903 \\ ROBERTO TRIGGIANI \\ Applied Mathematics, Thornton Hall \\ University of Virginia \\ Charlottesville, VA 22903
}

Key Words : Thermo-elastic plates, Analyticity of Semigroups, Uniform Decay Rates, Compactness of Semigroups, Essential Spectrum.

AMS Subject Qualification : 35, 93, 47.

\footnotetext{
${ }^{1}$ Research partially supported by the National Science Foundation under Grand DMS9504822, and by the Army Research Office under Grant DAAH04-96-1-0059.

Article published by EDP Sciences and available at http://www.edpsciences.org/proc or http://dx.doi.org/10.1051/proc:1998029
} 


\begin{abstract}
We consider (linear) thermo-elastic plate equations under five sets of canonical B.C., including two cases where the mechanical and the thermal variables are coupled on the boundary. The challenging so-called free B.C. case of $[\mathrm{Lag}],[\mathrm{A}-\mathrm{L}]$ is included. The main results are as follows. If rotational forces are not accounted for, then the resulting s.c. contraction semigroup is, moreover, analytic on the natural (energy) space under all such canonical B.C. By contrast, if rotational forces are accounted for, then the corresponding s.c. contraction semigroup has a structural property that makes it more akin to a s.c. group (at least in the mechanical part); a fortiori, it is neither compact, nor differentiable, nor uniformly continuous for all $t>0$. Analyticity of the s.c. thermo-elastic semigroup, particularly in the difficult case of free B.C., has been an open problem for some time in specialized circles. Similarly, a general description of the cases where analyticity fails has been the object of inquiries.
\end{abstract}




\section{Qualitative overview}

In this paper we focus on a preliminary fundamental issue concerning (linear) thermo-elastic plate equations, and its control theoretic consequences for associated optimal control problems: analyticity, or lack thereof, of the corresponding s.c. contraction semigroup (on the natural energy space). Despite extensive mathematical studies on thermo-elastic equations, the analyticity issue has been largely unsettled and the object of inquiries. We provide here a rather complete theory on these questions. Thermo-elastic equations, whose physical models arise in the case of two-dimensional plate equations, couple an elastic equation with the heat equation, thereby entailing an exchange of mechanical and thermal energy. One may associate with them five canonical sets of mechanical/thermal B.C., which we order by the level of increasing difficulties, from the most amenable to the most challenging, Set \#1 through Set \#5. In the first three cases, the problems may be viewed mathematically as being posed on an arbitrary (smooth) bounded domain of $\mathbf{R}^{n}$. The last two cases, instead, involve boundary operators which are tuned to the two-dimensional model. More seriously, in the last two cases, \#4 and \#5, the mechanical variable and the thermal variable are coupled on the boundary. Moreover, the boundary operators are of high order (two and three for the free set of B.C., Set \#5). This coupling adds genuine new difficulties over the preceding three cases, as the boundary coupling cannot be handled by standard perturbation theory. Cases where different portions of the boundary satisfy different sets of B.C. are also included in our treatment, though not explicitly mentioned here.

Our overall results can qualitatively be stated as follows. In each of the five canonical B.C. cases, the s.c. contraction semigroup of the thermo-elastic equation on the natural (energy) space is, moreover, analytic there (Theorem 2.1), if the elastic equation is of Euler-Bernoulli type, and hence does not account for rotational forces ( $\gamma=0$ in Eqn. (1.1) below). A fortiori, for such Euler-Bernoulli case $(\gamma=0)$, these s.c. contraction semigroups are exponentially stable in the uniform operator topology of the energy space, as one can readily eliminate the imaginary axis from the spectrum of the corresponding infinitesimal generator. By contrast, if the elastic equation is of Kirchoff-type, and hence does account for rotational forces $(\gamma>0$ in Eqn. (1.1) below), then the corresponding s.c. contraction semigroup has a group-like structural property at least in the mechanical part; a fortiori, it is neither compact, nor differentiable, nor uniformly continuous for $t>0$ - let alone analytic - under a general setting (Section 3), In addition, under the most amenable set of B.C., Set \#1, a rich spectral theory is available which in particular shows that there exists an infinite-dimensional invariant subspace, where the s.c. semigroup restricts to a group. However, even in the Kirchoff case $\gamma>0$, thermo-elastic equations are uniformly stable as well, a result which can be established by energy methods. Further comments and references to the literature are given after introducing the equations.

In the cases where analyticity of the s.c. semigroup holds true (EulerBernoulli, $\gamma=0$ ), it is possible to set up attractive boundary control/boundary observation optimal control problems, and show that they fit into established 
abstract (parabolic) theory for analytic semigroups, including a thorough treatment of the corresponding Riccati equations; see Section 4.

\subsection{P.D.E. models and literature}

P.D.E. models. The partial differential equations (P.D.E.'s) of linear thermoelastic plate equations on a bounded domain $\Omega$ of $\mathbf{R}^{2}$ are derived e.g., in Lagnese [Lag.1] and references therein. In general, a thermo-elastic system consists of an elastic equation in $w$ and a heat equation in $\theta$, which transfer mechanical and thermal energy through coupling. In the linear, homogeneous case, if one strips these equations from lower-order terms and normalizes the non-critical constants to 1 , they may be written as

$$
\left\{\begin{aligned}
w_{t t}-\gamma \Delta w_{t t}+\Delta^{2} w+\Delta \theta & =0 \text { in }(0, T] \times \Omega \equiv Q ; \\
\theta_{t}-\Delta \theta-\Delta w_{t} & =0 \text { in } Q
\end{aligned}\right.
$$

to be augmented by boundary conditions (B.C.) on $\partial \Omega$, and initial conditions $\left\{w_{0}, w_{1}, \theta_{0}\right\}$ at $t=0$. Here, the mechanical variable $w$ denotes the vertical displacement, while the thermal variable $\theta$ denotes the relative temperature about the stress-free state $\theta=0$. Moreover, $\gamma \geq 0$ is a constant. It is critical to distinguish between the case $\gamma=0$ (whereby (1.1) becomes the Euler-Bernoulli equation, with infinite speed of propagation) and the case $\gamma>0$ (whereby (1.1) becomes the hyperbolic Kirchoff equation with finite speed of propagation). The constant $\gamma$ accounts for rotational inertia and is proportional to the square of the thickness in the two-dimensional case. If one substitutes $\Delta \theta$ from (1.2) into (1.1), one obtains a second-order equation in $w$ which - formally and heuristically - has 'structural damping' [C-T.1-2] for $\gamma=0$, and 'viscous-type damping' for $\gamma>0$. The results in Section 2 and 3 below make these preliminary considerations precise.

Boundary Conditions. We associate with (1.1), (1.2) an appropriate set of mechanical and thermal B.C. The following five sets of B.C. are canonical.

(B.C. \#1) (Hinged mechanical/Dirichlet thermal B.C.) These are, in their simplest form, as follows:

$$
\left.w\right|_{\Sigma} \equiv 0 ;\left.\Delta w\right|_{\Sigma} \equiv 0 ;\left.\theta\right|_{\Sigma} \equiv 0, \Sigma=(0, T] \times \Gamma,
$$

with a refinement of the second one to the physical bending moment in $\operatorname{dim} \Omega=$ 2:

$$
\left.w\right|_{\Sigma}=0 ;\left[\Delta w+B_{1} w\right]_{\Sigma} \equiv 0 ;\left.\theta\right|_{\Sigma} \equiv 0, \text { on } \Sigma,
$$

where $B_{1}$ is the boundary operator defined in (1.7d) below.

(B.C. \#2) (Clamped mechanical/Dirichlet thermal B.C.) These are

$$
\left.w\right|_{\Sigma} \equiv 0 ;\left.\frac{\partial w}{\partial \nu}\right|_{\Sigma} \equiv 0 ;\left.\theta\right|_{\Sigma} \equiv 0 \text { on } \Sigma .
$$


(B.C. \#3) (Clamped mechanical/Neumann (Robin) thermal B.C.) These are

$$
\left.w\right|_{\Sigma} \equiv 0,\left.\frac{\partial w}{\partial \nu}\right|_{\Sigma} \equiv 0 ;\left[\frac{\partial \theta}{\partial \nu}+b \theta\right]_{\Sigma}=0 ; b \geq 0
$$

(B.C. \#4) (Hinged mechanical/Neumann (Robin) thermal B.C.) These are

$$
\left.w\right|_{\Sigma} \equiv 0 ;\left[\Delta w+B_{1} w+\theta\right]_{\Sigma}=0 ;\left[\frac{\partial \theta}{\partial \nu}+b \theta\right]_{\Sigma}=0 . b \geq 0,
$$

where $B_{1}$ is the same boundary operator as in $(1.3 \mathrm{~b})$, and is defined in $(1.7 \mathrm{~d})$ below. 0 :

(B.C. \#5) (Free mechanical/Robin thermal B.C.) These are for $\gamma=$

$$
\left\{\begin{array}{l}
{\left[\Delta w+B_{1} w+\theta\right]_{\Sigma}=0} \\
{\left[\frac{\partial \Delta w}{\partial \nu}+B_{2} w-w+\frac{\partial \theta}{\partial \nu}\right]_{\Sigma}=0 ;\left[\frac{\partial \theta}{\partial \nu}+b \theta\right]_{\Sigma}=0, \quad b \geq 0}
\end{array}\right.
$$

where the boundary operators $B_{1}$ and $B_{2}$ are explicitly given by [Lag.1] with $0 \leq \mu<1$ :

$$
\text { on } \begin{aligned}
\Sigma: B_{1} w & =(1-\mu)\left[2 \nu_{1} \nu_{2} w_{x y}-\nu_{1}^{2} w_{y y}-\nu_{2}^{2} w_{x x}\right] ; \\
B_{2} w & =(1-\mu) \frac{\partial}{\partial \tau}\left[\left(\nu_{1}^{2}-\nu_{2}^{2}\right) w_{x y}+\nu_{1} \nu_{2}\left(w_{y y}-w_{x x}\right)\right] .
\end{aligned}
$$

We note explicitly that B.C. Sets \#4 and \#5 include a coupling between the mechanical and the thermal variables $w$ and $\theta$. The above sets are listed in the order of 'increasing difficulty' in the analysis leading to Theorem 2.1 below. One may also consider different sets of B.C. on different portions of the boundary, see one such example in [L-T.1, Example 5.5].

Literature. This is by no means the place for a review on the vast literature on thermo-elasticity in general. Rather, we shall primarily concentrate on the issues where the present paper announces new results: (i) analyticity of the s.c. contraction semigroup arising from the thermo-elastic plate equations (1.1), (1.2) for $\gamma=0$, under various B.C.; (ii) by contrast, the structural property: "group (at least in the mechanical variables) plus a 'differentiable' perturbation," for (1.1), (1.2) with $\gamma>0$. These two issues will be embedded in the problem of stability of thermo-elastic systems: this, historically, has been the object of intense investigations which preceded, in fact, analyticity; see below.

Case $\gamma=0$. Following the numerous works on stability (below), it was only recently that a much stronger, and more desirable, result was established in [LR.1], at least for one set of B.C., via a technical proof: that in the case of B.C. 
\#2 of clamped B.C. for $w /$ Dirichlet B.C. for $\theta$, the associated s.c. contraction semigroup is, in fact, analytic. This is the first serious result on analyticity. Analyticity for B.C. Set \#1, (1.3a) is a rather easy matter [L-R.1], [R.1], with the case of B.C. Set \#1, (1.3b) being then treated as a perturbation of (1.3a) [T.1]. Henceforth B.C. Set \#1 is considered only for completeness; actually, a rich spectral theory for both cases $\gamma>0$ and $\gamma=0$ is available in the case of B.C. Set \#1, (1.3a), see [Cg-T.1], going much beyond analyticity of the semigroup for $\gamma=0$ and lack of analyticity for $\gamma>0$. Once analyticity is established, it is not difficult to infer that the semigroup is also uniformly stable, by excluding the possibility that the generator has spectrum on the imaginary axis, see details in [L-T.5, Chapter 3]. It is plainly desirable to have an abstract setting and an abstract proof of analyticity, which covers and encompasses at least several sets of physical B.C. Such goal is achieved in both recent papers [L-L.1] and [L-T.1], independently. The proof in [L-L.1] is by a contradiction argument: it assumes that the well-known characterization of analyticity based on the resolvent of the generator is violated and gets a contradiction. Generally, direct proofs are more desirable than proofs by contradiction, in that the former provide more insight than the latter. In the present case in question, direct proofs have the advantage of admitting semi-discrete counterparts, of interest in the numerical analysis of these equations, with control of the constants arising in the relevant estimates. In [L-T.1], two direct proofs of analyticity of the underlying s.c. contraction semigroup are provided, which arises from abstract thermoelastic systems of Euler-Bernoulli type: $\gamma=0$, under slightly different sets of assumptions. Though differently conceived, both abstract frameworks in [LL.1] and [L-T.1] do not cover B.C. Sets \#4 and \#5. [The only thermo-elastic analytic plate example of [L-L.1] is readily covered by [L-T.1, Example 5.5], as well.] Therefore, [L-T.2] pushes further the analysis, by injecting, in addition, ad hoc P.D.E. estimates for the specific cases (in contrast with the rather abstract analysis of [L-T.1]), and thus succeeds in establishing analyticity for the B.C. \#4 and \#5 as well. The proofs are lengthy and technical, particularly for the most demanding case of B.C. \#5; a sketch is provided in Section 2.2.

Case $\gamma>0$. If the mechanical equation accounts for rotational forces, $\gamma>0$ in (1.1), and thus becomes the Kirchoff equation rather than the Euler-Bernoulli equation, it was pointed out in [T.1] that the corresponding s.c. semigroup of contraction fails to be analytic, at least for the most amenable B.C., Set \#1 (hinged). A richer theory for this B.C. Set \#1, (1.3a) is available, which shows much more $[\mathrm{Cg}-\mathrm{T}]$ : in particular, there exists an infinite dimensional invariant subspace where the s.c. semigroup restricts to a group. This analysis expands on the results obtained in [H.1] in the case of a one-dimensional thermo-elastic rod, where the eigenvalues approach asymptotically a vertical line. See also [H-Z.1]. Under all canonical B.C., Set \#2 through Set \#5, thus including the coupled B.C. cases, a recent paper [L-T.3], which is devoted to the case $\gamma>0$, shows that the s.c. thermo-elastic plate semigroup is the sum of a s.c. group in the mechanical variables plus a (possibly, fractionally) differentiable perturbation. A fortiori, the thermo-elastic plate semigroup for $\gamma>0$ is neither compact, 
nor differentiable, nor uniformly continuous in the uniform operator topology for $t>0$; let alone analytic. A related result with the semigroup decomposed as a simpler semigroup plus a compact perturbation, was previously shown in [H-L-P.1], for an abstract system, which is however motivated by $n$-dimensional systems of thermo-elasticity. When applied to thermo-elastic plate equations such as (1.1), (1.2), only the case of hinged/Dirichlet B.C., Set \#1, is covered. Already at the level of clamped/Dirichlet B.C., Set \#2, the abstract assumption (H.1) in [H-L-P.1, p. 67] fails to hold true (because of compatibility conditions). In this latter case of Set \#2 the same decomposition result as in [H-L-P.1] was later proved in [T-Z.1], as an adaptation of [H-L-P.1]. This Set \#2 case is also a special case of the more general results in [L-T.3], where the decomposition: group in the mechanical variables plus a 'differentiable' perturbation, is established also for the most demanding Sets \#4 and \#5 of coupled B.C. A decomposition method for integro-partial differential equations was given also in [Leu.1].

Regarding the issue of stability, see a detailed literature overview with a comprehensive list of references in [Las.1], [L-R.1], [L-L.1]. This means that heat dissipation alone is sufficiently strong to induce exponential energy decay. At first, and for some time, thermo-elastic plate equations were the object of successful studies showing asymptotic exponential stability of their solutions, initially under additional mechanical dissipation in the free B.C. case and actually for $\gamma>0$ [Lag.1]; later for $\gamma=0$, in several papers, under homogeneous B.C. of different type: e.g., [K.1] for B.C. Set \#2; [S.1] for B.C. Set \#3; [L-Z.1], etc.; see also the simpler case of a $\operatorname{rod}(\operatorname{dim} \Omega=1)$ in [B-L-Z], [H.1]. As already mentioned, exponential decay for $\gamma=0$ is then, a fortiori, a consequence of the much stronger result of analyticity of the corresponding semigroup. However, asymptotic exponential stability of the solutions of thermo-elastic plate equations with $\gamma>0$ continues to remain true under various sets of B.C. For the first three cases, uniform stability is established in [A-L.1] for all $\gamma \geq 0$, with bounds independent of $\gamma \geq 0$, by virtue of a novel operator multiplier technique, which yields sharp results. This same operator multiplier technique was subsequently used in [A-L.2] to prove exponential stability of the thermo-elastic plate solutions of (1.1), (1.2), under the most demanding free B.C. (Set \#5), for $\gamma>0$. Analyticity of the semigroup for $\gamma=0$, claimed in our Theorem 2.1 below for free B.C., provides a-fortiori also exponential stability in this case $\gamma=0$, thus complementing [A-L.2] for $\gamma>0$. The same critical operator multiplier technique of [A-L.1], [A-L.2] was later used also in [PM-Z.1] in obtaining the same results of [A-L.1], this time for a von Karman thermo-elastic plate equation with $\gamma \geq 0$, clamped/Dirichlet B.C. (Set \#2); and in [A-L.3] for a larger class of non-linearities. The power of the $[\mathrm{A}-\mathrm{L}]$-operator multiplier technique has been recognized in the introduction of [PM-Z.1], where these authors make a comparison with the following much weaker results that they had previously obtained in a joint effort with two other authors, E. Bisognin and V. Bisognin, referenced in [PM-Z.1]: (i) the case $\gamma>0$ was handled by the structural property of the semigroup (simpler semigroup plus a compact perturbation) from [T-Z.1] (as referred to above), yielding decay rates which blow-up with $\gamma \searrow 0$; (ii) for this 
reason, the case $\gamma=0$ had to be handled separately, by invoking the analyticity of the semigroup from [L-R.1].

These stability results on thermo-elastic plates followed, to be sure, prior investigations on the stability of $n$-dimensional systems of thermo-elasticity, the precursor of which is a strong stability result (and a generation of a s.c. contraction semigroup result) in [D.1]. In the case $n=1$, exponential stability was shown in [L-Z.1] under different B.C. in the energy space if the equation has constant coefficients; and previously in [Sl.1] in a higher norm, if the equation has variable coefficients. The stability issue is re-examined in [H-L-P.1], in terms of the aforementioned decomposition property, for the $n$-dimensional system of thermo-elasticity. In [H-L-P.1, Corollaries 4 and 5], it is established that exponential decay holds true if $n=1$, thus recovering [L-Z.1], [Sl.1]; however, it fails to hold true for $n \geq 2$, at least in the case of periodic B.C. It is further conjectured in [H-L-P.1, Remark p. 70], that failure of exponential decay for $n \geq 2$ should attain also in the case of Dirichlet B.C. This conclusion was then shown to be true in [L-Z.1], at least for some classes of domains in $\mathbf{R}^{3}$ which include convex domains, by also employing the decomposition of [H-L-P.1] and geometric optics techniques. These negative results on exponential stability for $n$-dimensional systems of elasticity for $n \geq 2$, should be contrasted with the positive results on exponential stability for the plate system (1.1), (1.2) under all canonical B.C., discussed above.

Regarding the problem of controllability, we briefly mention some recent references. For a problem of type (1.1), (1.2) except with sufficiently small coupling between the mechanical and the thermal components; with $\gamma>0$, and with two boundary controls (moments and shears), an exact controllability result only for the mechanical part is given in [Lag.2]. Exact controllability results for the displacement and simultaneously approximate controllability results for the thermal component are given in: (i) [Z.1] and [T-Z.1], for a 3-dimensional system of elasticity, respectively for problem (1.1), (1.2) with $\gamma>0$ and clamped/Dirichlet B.C. (Set \#2), in both cases with mechanical distributed control (i.e., acting on the mechanical equation) and supported in a neighborhood of the boundary; (ii) and in [A.1] for problem (1.1), (1.2) with $\gamma \geq 0$, with clamped/Dirichlet B.C. (Set \#2), and with thermal distributed control (i.e., acting on the thermal equation, and thus in need of transferring its influence to the mechanical equation via coupling). Reference [A.1] again employs the operator multiplier technique as in [A-L.1]-[A-L.3]. Regarding exact null-controllability, we quote [H-Z.1] in the case of a one-dimensional system (1.1), (1.2) with hinged/Dirichlet B.C.; with just one boundary control, either mechanical or thermal (with precise results obtained via an associated moment problem, a technique which does not admit an extension to higher dimension); and [L-T.6] for the multidimensional case, with $\gamma=0$, hinged/Dirichlet B.C. (Set \#1) and distributed control, either mechanical or thermal. 


\subsection{Basic well-posedness: Generation of a s.c. contraction semigroup}

Well-posedness of problem (1.1), (1.2) under any set, \#1 through \#5, of B.C. can be readily settled within the framework of Lumer-Phillips Theorem, to yield

Theorem 1.1. With reference to problem (1.1), (1.2) with $\gamma \geq 0$ and any set of B.C.—\#1, (1.3a), \#2 through \#5-the map: $\left\{w_{0}, w_{1}, \theta_{0}\right\} \rightarrow\left\{w(t), w_{t}(t), \theta(t)\right\}$, from the initial condition to the solution, defines a s.c. (strongly continuous) contraction semigroup for $t \geq 0$, on a 'natural' (energy) space (see below), which depends on the specific B.C. and on $\gamma>0$ or $\gamma=0$ (see below). B.C. (1.3b) can be handled as a perturbation of B.C. (1.3a).

The proof is rather straightforward for B.C. \#1, (1.3a) through \#3, and a bit more elaborate for B.C. \#4 and \#5, see e.g., [L-T.5, Chapter 3]. Examples of 'natural' spaces will be given below.

The issue of interest in the present paper is: when is the s.c. contraction semigroup guaranteed by Theorem 1.1, moreover, analytic? For this, we have to critically distinguish between the case $\gamma=0$ and the case $\gamma>0$. An affirmative answer implies, in particular, the property that the s.c. semigroup is exponentially stable in the uniform operator topology of the energy space, as one can readily exclude the imaginary axis from the spectrum of the infinitesimal generator. Thus, Section 2 below recovers the above-quoted results of the literature, where uniform stability of the solutions (in the energy space) was obtained. Moreover, it also includes a new case: uniform stability for $\gamma=0$ under free B.C., Set \#5.

\section{Case $\gamma=0$ : Analyticity of the semigroup}

Let the constant $\gamma=0$ in Eqn. (1.1). Then, the corresponding semigroup is analytic.

Theorem 2.1. With reference to the thermo-elastic Eqns. (1.1), (1.2) with $\gamma=0$, the s.c. semigroup guaranteed by Theorem 1.1 is, moreover, analytic on the natural (energy) space [below], under each set of B.C., \#1 through \#5. Moreover, such semigroup is exponentially stable in the uniform operator topology.

Remark 2.1. It is plainly desirable to have an abstract setting and an abstract proof of analyticity, which covers and encompasses at least several sets of physical B.C. The abstract setting given below in Section 2.1, after [LT.1], encompasses, in particular, B.C. Sets \#1, \#2, and \#3 (in addition to other examples, see [L-T.1, Section 5]). However, B.C. Sets \#4 and \#5 (which couple the mechanical and the thermal variables $w$ and $\theta$ ) are excluded from it. Thus, in the case of B.C. \#1, \#2, \#3, the validity of Theorem 2.1 follows from

ESAIM: Proc., Vol. 4, 1998, 199-222 
Theorem 2.2 below on the abstract model (2.11). Instead, a sketch of the proof of Theorem 2.1 in the case of the more challenging B.C. \#4 and \#5 (which do not fit the abstract model (2.11)) is relegated to Section 2.2.

\subsection{Abstract setting for B.C. Sets \#1, (1.3a); \#2; and \#3}

Mathematical setting. Let $X$ be a Hilbert space with norm \|\|$_{X}$ and inner product $(,)_{X}$. On it, we consider two operators $\mathcal{A}$ and $\mathcal{B}$ subject to the following three sets of assumptions.

Set \#1. It consists of assumptions (H.1), (H.2) [or (H.2 weak)], and (H.3) below.

(H.1) $\mathcal{A}: X \supset \mathcal{D}(\mathcal{A}) \rightarrow X$ and $\mathcal{B}: X \supset \mathcal{D}(\mathcal{B}) \rightarrow X$ are two strictly positive self-adjoint operators;

$$
\mathcal{D}\left(\mathcal{A}^{\frac{1}{2}}\right) \subset \mathcal{D}(\mathcal{B}), \text { equivalently } \mathcal{B} \mathcal{A}^{-\frac{1}{2}} \in \mathcal{L}(X)
$$

(the implications $\Rightarrow$ follows by the closed graph theorem);

(H.3) there is a constant $c>0$ such that

$$
c\left\|\mathcal{A}^{\frac{1}{4}} x\right\|_{X} \leq\left\|\mathcal{B}^{\frac{1}{2}} x\right\|_{X}, \quad \forall x \in \mathcal{D}\left(\mathcal{A}^{\frac{1}{4}}\right) .
$$

Remark 2.2. By Lowner's Theorem [K.2, Corollary 7.1, p. 146], condition (2.1) of assumption (H.2) implies:

(H.2w)

$$
\begin{array}{r}
\mathcal{D}\left(\mathcal{A}^{\frac{1}{4}}\right) \subset \mathcal{D}\left(\mathcal{B}^{\frac{1}{2}}\right), \text { equivalently } \mathcal{B}^{\frac{1}{2}} \mathcal{A}^{-\frac{1}{4}} \in \mathcal{L}(X), \\
\text { equivalently }\left\|\mathcal{B}^{\frac{1}{2}} x\right\|_{X} \leq C\left\|\mathcal{A}^{\frac{1}{4}} x\right\|_{X}, \quad \forall x \in \mathcal{D}\left(\mathcal{A}^{\frac{1}{4}}\right) .
\end{array}
$$

Thus, assumption $($ H.3 $)=(2.2)$ reverses inequality (2.3) - which is implied by assumption (H.2) in (2.1) -however, in a relaxed form; i.e., only for smoother elements $x \in \mathcal{D}\left(\mathcal{A}^{\frac{1}{4}}\right)$, not necessarily for all $x \in \mathcal{D}\left(\mathcal{B}^{\frac{1}{2}}\right)$. Thus, (2.2) is generally weaker than the requirement $\mathcal{D}\left(\mathcal{B}^{\frac{1}{2}}\right) \subset \mathcal{D}\left(\mathcal{A}^{\frac{1}{4}}\right)$. This distinction is important in the case of B.C. Set \#3 (clamped B.C. on the mechanical variable; Neumann B.C. on the thermal variable). Notice that (H.1), (H.2w), (H.3) yield that $\mathcal{A}^{-\frac{1}{4}} \mathcal{B} \mathcal{A}^{-\frac{1}{4}}$ is a bounded, boundedly invertible, self-adjoint operator on $\mathcal{L}(X)$.

Set \#2. It consists of assumption (H.1) above, as well as assumptions (A.2), (A.3) below.

$$
\mathcal{D}(\mathcal{B}) \subset \mathcal{D}\left(\mathcal{A}^{\frac{1}{2}}\right)
$$

$$
\mathcal{D}\left(\mathcal{B}^{\frac{1}{2}}\right)=\mathcal{D}\left(\mathcal{A}^{\frac{1}{4}}\right)
$$


Thus, a-fortiori, (A.3) implies both $($ H.2w) $=(2.3)$ and $($ H.3 $)=(2.2)$ above.

Set \#3. (Generalizing Set \#1) It consists of assumptions (H.1), (H.2) = (2.1) above, as well as of assumption (SD) [for "structural damping"] below:

(SD) for constants $\frac{1}{2} \leq \alpha \leq 1$, and $0<c<C<\infty$, we have:

$$
c\left\|\mathcal{A}^{\frac{\alpha}{2}} x\right\|_{X} \leq\left\|\mathcal{B}^{\frac{1}{2}} x\right\|_{X} \leq C\left\|\mathcal{A}^{\frac{\alpha}{2}} x\right\|, \quad \forall x \in \mathcal{D}\left(\mathcal{A}^{\frac{\alpha}{2}}\right)
$$

equivalently,

$$
c^{2}\left(\mathcal{A}^{\alpha} x, x\right) \leq(\mathcal{B} x, x)_{X} \leq C^{2}\left(\mathcal{A}^{\alpha} x, x\right) \quad \forall x \in \mathcal{D}\left(\mathcal{A}^{\frac{\alpha}{2}}\right) .
$$

A sufficient condition for (2.6) to hold is

$$
\mathcal{D}\left(\mathcal{B}^{\frac{1}{2}}\right)=\mathcal{D}\left(\mathcal{A}^{\frac{\alpha}{2}}\right) .
$$

For $\alpha=\frac{1}{2}$, assumption (2.6) reduces to the combination of $(\mathrm{H} .2 w)=(2.3)$ and $(\mathrm{H} .3)=(2.2)$ together. Thus, Set \#3 generalizes Set \#1 to which it reduces when $\alpha=\frac{1}{2}$. Notice that (H.1) and (SD) yield that $\mathcal{A}^{-\frac{\alpha}{2}} \mathcal{B} \mathcal{A}^{-\frac{\alpha}{2}}$ is a bounded, boundedly invertible, self-adjoint operator in $\mathcal{L}(X)$.

Abstract thermo-elastic system. The abstract thermo-elastic system considered in this subsection is

$$
\left\{\begin{aligned}
w_{t t}+\mathcal{A} w-\mathcal{B} \theta & =0, \\
\theta_{t}+\mathcal{B} \theta+\mathcal{B} w_{t} & =0,
\end{aligned}\right.
$$

or, in first-order form, with $y(t)=\left[w(t), w_{t}(t), \theta(t)\right]$,

$$
\begin{gathered}
\dot{y}=A y ; \quad y(0)=y_{0}=\left[w_{0}, w_{1}, \theta_{0}\right] \in Y ; \\
A=\left[\begin{array}{rrr}
0 & I & 0 \\
-\mathcal{A} & 0 & \mathcal{B} \\
0 & -\mathcal{B} & -\mathcal{B}
\end{array}\right]: Y \supset \mathcal{D}(A) \rightarrow Y ; \\
A^{*}=\left[\begin{array}{rrr}
0 & -I & 0 \\
\mathcal{A} & 0 & -\mathcal{B} \\
0 & \mathcal{B} & -\mathcal{B}
\end{array}\right] ; \mathcal{D}\left(A^{*}\right)=\mathcal{D}(A),
\end{gathered}
$$

where $A^{*}$ is the $Y$-adjoint of $A$; moreover,

$$
Y=\mathcal{D}\left(\mathcal{A}^{\frac{1}{2}}\right) \times X \times X ; \mathcal{D}(A)= \begin{cases}\mathcal{D}(\mathcal{A}) \times \mathcal{D}\left(\mathcal{A}^{\frac{1}{2}}\right) \times \mathcal{D}(\mathcal{B}), & \text { under }(\mathrm{H} .2) \\ \mathcal{D}(\mathcal{A}) \times \mathcal{D}(\mathcal{B}) \times \mathcal{D}(\mathcal{B}), & \text { under }(\mathrm{A} .2)\end{cases}
$$

As a straight-forward application of the Lumer-Phillips theorem [P.1, p. 14] directly, or else of its standard corollary [P.1, p. 15] involving $A$ and $A^{*}$, one 
readily proves that: $A$ and $A^{*}$ in (2.11) are maximal dissipative and generate s.c. contraction semigroups $e^{A t}$ and $e^{A^{*} t}$ on $Y$.

The main abstract result of the present subsection is:

Theorem 2.2. Assume either one of the three sets of assumptions: either Set \#1 [(H.1), (H.2) = (2.1), (H.3) = (2.2)], or else Set \#2 [(H.1), (A.2) = (2.4), (A.3) = (2.5)], or else Set \#3 [(H.1), (H.2) $=(2.1),(S D)=(2.6)]$. Then, the resolvent $R(\lambda, A)$ of $A$ in (2.11) satisfies the estimate

$$
\|R(\lambda, A)\|_{\mathcal{L}(Y)} \leq \frac{C}{|\lambda|}, \quad \forall \lambda \text { with } \operatorname{Re} \lambda>0 .
$$

Hence, A generates a s.c. contraction semigroup which, moreover, is analytic (holomorphic) on $Y, t>0$ [F.1, p. 180-185].

Comments about the proofs of Theorem 2.2. Concerning Theorem 2.2, the authors have obtained the following three direct proofs:

(i) two proofs [L-T.1] critically exploit the results of [C-T.1-2] on structurally damped second-order equations, which state (in particular) that: the operator

$$
\begin{gathered}
-A_{1}=\left[\begin{array}{rr}
0 & I \\
-\mathcal{A} & -\mathcal{B}
\end{array}\right]: Y_{1} \supset \mathcal{D}\left(A_{1}\right) \rightarrow Y_{1} ; \\
Y_{1}=\mathcal{D}\left(\mathcal{A}^{\frac{1}{2}}\right) \times X ; \mathcal{D}\left(A_{1}\right)= \begin{cases}\mathcal{D}(\mathcal{A}) \times \mathcal{D}\left(\mathcal{A}^{\frac{1}{2}}\right), & \text { under (H.2); } \\
\mathcal{D}(\mathcal{A}) \times \mathcal{D}(\mathcal{B}), & \text { under (A.2). }\end{cases}
\end{gathered}
$$

generates a s.c. analytic semigroup on the space $Y_{1}$, under the stated assumptions. These two proofs are, however, quite different from each other; one uses Set \#1 and Set \#2 of assumptions, the other Set \#3 of assumptions.

Proof \#1. One proof couples $\left[w, w_{t}\right]$ with $\theta$ and exploits the energy dissipation of $\theta$. More precisely, after substituting $\mathcal{B} \theta$ from (2.9) into (2.8), the original abstract system (2.8), (2.9) may be alternatively rewritten as

$$
w_{t t}+\mathcal{A} w+\mathcal{B} w_{t}=-\theta_{t} ; \quad \text { or } \frac{d}{d t}\left[\begin{array}{c}
w \\
w_{t}
\end{array}\right]=-A_{1}\left[\begin{array}{c}
w \\
w_{t}
\end{array}\right]-\left[\begin{array}{c}
0 \\
\theta_{t}
\end{array}\right],
$$

where the operator $\left(-A_{1}\right)$ is defined by $(2.14)$. Consideration at the outset of (2.17) in place of $(2.8),(2.9)$ is very natural, as remarked in the paragraph below (1.2). A very different decomposition is used in [H-L-P.1, Eqn. (9)] for the $n$-dimensional system of elasticity (which however is not analytic; it rather corresponds to (1.1), (1.2) for $\gamma>0)$. Thus, our proof proceeds along quite different lines from [H-L-P.1]. On the strength of assumptions (H.1), (H.2w) = $(2.1),($ H.3 $)=(2.2),\left(-A_{1}\right)$ is the generator of a s.c. analytic semigroup $e^{-A_{1} t}$ on the space $Y_{1}$ defined by (2.15), (2.16) [C-T.1-2]. Notice that $Y \equiv Y_{1} \times X$, 
see (2.12). Thus, the Laplace transform solution of $(2.17)$, initiating at $y_{0}=$ $\left[w_{0}, w_{1}, \theta_{0}\right]$, is

$$
\left[\begin{array}{c}
\hat{w}(\lambda) \\
\hat{w}_{t}(\lambda)
\end{array}\right]=R\left(\lambda,-A_{1}\right)\left[\begin{array}{c}
w_{0} \\
w_{1}
\end{array}\right]+R\left(\lambda,-A_{1}\right)\left[\begin{array}{c}
0 \\
\theta_{0}
\end{array}\right]-R\left(\lambda,-A_{1}\right)\left[\begin{array}{c}
0 \\
\lambda \hat{\theta}(\lambda)
\end{array}\right] .
$$

Eqn. (2.18) expresses the mechanical variables $\left\{w, w_{t}\right\}$ in terms of the thermal variable $\theta$. On the other hand, the following energy dissipation inequality holds true:

$$
\left\|\mathcal{B}^{\frac{1}{2}} \hat{\theta}(\lambda)\right\|_{X}^{2} \leq C\left\|\mathcal{B}^{-\frac{1}{2}} \theta_{0}\right\|_{X}^{2}+\left|\left(\left[\begin{array}{c}
w_{0} \\
w_{1}
\end{array}\right],\left[\begin{array}{c}
\hat{w}(\lambda) \\
\hat{w}_{t}(\lambda)
\end{array}\right]\right)_{Y_{1}}\right|
$$

Eqn. (2.19) expresses the thermal variable $\theta$ in terms of the mechanical variables $\left\{w, w_{t}\right\}$. To uncouple (2.18) and (2.19), one begins by applying $A_{1}^{\frac{1}{2}}$ to $(2.18)$ and by using analyticity estimates for the resolvent of $\left(-A_{1}\right)$. Details are provided in [L-T.1].

Proof \#2. This proof is purely operator theoretic. It transforms the original thermo-elastic generator $A$ in (2.11) into a more amenable form, by means of an explicitly constructed similarity transformation. Modulo innocuous perturbations of the identity operator and modulo a serious perturbation which is handled by the estimates of [C-T.1-2], the thermo-elastic generator $A$ in (2.11) is similar to the block-diagonal operator

$$
A_{d}=\left[\begin{array}{ccc}
0 & \frac{1}{2} I & 0 \\
-\mathcal{A} & \lambda_{1} \mathcal{B} & 0 \\
0 & 0 & \lambda_{2} \mathcal{B}
\end{array}\right], \quad \operatorname{Re} \lambda_{i}<0
$$

whereby analyticity of the semigroup generated by $A_{d}$ in $(2.20)$ follows via [CT.1-2], on the strength of Set \#3 of assumptions. The construction to obtain $A_{d}$ in (2.20) from $A$ in $(2.11)$ begins by diagonalizing the $2 \times 2$ block $\left[\begin{array}{cc}0 & \mathcal{B} \\ -\mathcal{B} & -\mathcal{B}\end{array}\right]$ in (2.11). Details are given in [L-T.1].

(ii) A third direct proof [L-T.2] is more flexible, and is based on transferring, on a term-by-term basis, the estimate coming from dissipativity of $\theta$, to other terms of the resolvent of $A$, in a suitable sequence. These ideas will be expanded in subsection 2.2 below, where, with the addition of P.D.E. estimates pertaining to the basic elastic and thermal operators, provide a direct proof of analyticity in the case of the most challenging B.C. \#4 and \#5.

Applications of Theorem 2.2 to B.C. Set \#1, \#2, and \#3. B.C. Set \#1, (1.3a). Theorem 2.2 applies to this case, with $X=L_{2}(\Omega)$ and $\mathcal{B}=\mathcal{A}^{\frac{1}{2}}$, where

$$
\mathcal{A}=\Delta^{2}, \mathcal{D}(\mathcal{A})=\left\{f \in H^{4}(\Omega):\left.f\right|_{\Gamma}=\left.\Delta f\right|_{\Gamma}=0\right\}
$$

ESAIM: Proc., Vol. 4, 1998, 199-222 
B.C. Set \#2, (1.4). Theorem 2.2 applies to this case with (i)

$$
X \equiv L_{2}(\Omega) ; \mathcal{A} f=\Delta^{2} f, \mathcal{D}(\mathcal{A})=\left\{f \in H^{4}(\Omega):\left.f\right|_{\Gamma}=\left.\frac{\partial f}{\partial \nu}\right|_{\Gamma} \equiv 0\right\}
$$

(ii)

$$
\mathcal{B}=\mathcal{A}_{D}, \text { where } \mathcal{A}_{D} f=-\Delta f ; \mathcal{D}\left(\mathcal{A}_{D}\right)=H^{2}(\Omega) \cap H_{0}^{1}(\Omega) .
$$

Then, $\mathcal{A}$ and $\mathcal{B}$ are positive, self-adjoint operators on $L_{2}(\Omega)$, and (H.1) is verified. Moreover,

$$
\begin{gathered}
\mathcal{D}\left(\mathcal{A}^{\frac{1}{2}}\right) \equiv H_{0}^{2}(\Omega) \subset \mathcal{D}(\mathcal{B}) \equiv H^{2}(\Omega) \cap H_{0}^{1}(\Omega) ; \\
\mathcal{D}\left(\mathcal{A}^{\frac{1}{4}}\right) \equiv H_{0}^{1}(\Omega)=\mathcal{D}\left(\mathcal{B}^{\frac{1}{2}}\right)=\mathcal{D}\left(\mathcal{A}_{D}^{\frac{1}{2}}\right) .
\end{gathered}
$$

Thus, $(2.24)$ verifies $($ H.2) $=(2.1)$; while $(2.25)$ is stronger than $($ H.3 $)=(2.2)$.

B.C. Set \#3, (1.5). Theorem 2.2 applies to this case with $X=L_{2}(\Omega), \mathcal{A}$ as in $(2.22)$ and

$$
\mathcal{B}=\mathcal{A}_{N} ; \mathcal{A}_{N} f=-\Delta f, \mathcal{D}\left(\mathcal{A}_{N}\right)=\left\{f \in H^{2}(\Omega):\left[\frac{\partial f}{\partial \nu}+b f\right]_{\Gamma} \equiv 0\right\} .
$$

Then

$$
\mathcal{D}\left(\mathcal{A}^{\frac{1}{2}}\right) \equiv H_{0}^{2}(\Omega) \subset \mathcal{D}(\mathcal{B})=\mathcal{D}\left(\mathcal{A}_{N}\right),
$$

so that $(\mathrm{H} .2)=(2.1)$ is verified. Moreover,

$$
\left\|\mathcal{A}^{\frac{1}{4}} f\right\|_{X}=\left\|\mathcal{A}_{N}^{\frac{1}{2}} f\right\|_{X}=\left\|\mathcal{B}^{\frac{1}{2}} f\right\|_{X}=\left\{\int_{\Omega}|\nabla f|^{2} d \Omega\right\}^{\frac{1}{2}}, \forall f \in \mathcal{D}\left(\mathcal{A}^{\frac{1}{4}}\right) \subset \mathcal{D}\left(\mathcal{B}^{\frac{1}{2}}\right),
$$

and $($ H.3) $=(2.2)$ is verified.

Further examples are given in [L-T.1, Section 5], including a case of partially hinged/partially clamped mechanical B.C. with Dirichlet thermal B.C., which is the only analytic thermo-elastic example given in [L-L.1].

\subsection{The proofs of Theorem 2.1 for the B.C. Sets \#4 and \#5}

Here we can only limit ourselves to a meager sketch providing the incipient idea and the general strategy of the proofs. The actual proofs are technical and lengthy, particularly in the case of B.C. Set \#5, for which we refer to [L-T.2]. Let

$$
f_{0}=\left[u_{0}, v_{0}, \theta_{0}\right] \in Y \equiv \mathcal{D}\left(\mathcal{A}^{\frac{1}{2}}\right) \times L_{2}(\Omega) \times L_{2}(\Omega) ;
$$




$$
\mathcal{D}\left(\mathcal{A}^{\frac{1}{2}}\right)= \begin{cases}\mathcal{D}\left(\mathcal{A}_{D}\right)=H^{2}(\Omega) \cap H_{0}^{1}(\Omega), & \text { for B.C. Set \#4; } \\ H^{2}(\Omega), & \text { for B.C. Set \#5. }\end{cases}
$$

If $A$ is the generator-which may be explicitly given, see e.g., [L-T.5, Chapter 3] - then let $\omega \in \mathbf{R}$, and define

$$
y(\omega)=[u(\omega), v(\omega), \theta(\omega)]=[i \omega I-A]^{-1} f_{0}=R(i \omega, A) f_{0} \in \mathcal{D}(A),
$$

where the resolvent operator of $A$ is well-defined on the imaginary axis, as one may readily exclude the imaginary axis from the spectrum of $A$, in both cases B.C. \#4 and \#5. Our goal is to verify the following resolvent characterization [for $A$ which is already known, see Theorem 1.1, to be the generator of a s.c. contraction semigroup]: there exists a constant $C>0$ s.t. for all $\omega \in \mathbf{R}$, with say $|\omega| \geq$ some $\omega_{0}>0$, we have

$$
\|[u(\omega), v(\omega), \theta(\omega)]\|_{Y}=\|y(\omega)\|_{Y}=\left\|R(i \omega, A) f_{0}\right\|_{Y} \leq \frac{C}{|\omega|}\left\|f_{0}\right\|_{Y} .
$$

Remark 2.3. To justify the above resolvent characterization in the present setting, we provide the following sketch, while referring to [L-T.5, Chapter 3, Appendix E, Theorem E.3] for technical details. (i) First, since $A$ is the generator of a s.c. contraction semigroup, it follows that $\|R(\lambda, A)\| \leq 1 / \operatorname{Re} \lambda$ for all $\lambda \in \mathbf{C}^{+} \equiv\{\lambda \in \mathbf{C}, \operatorname{Re} \lambda>0\}$; thus a fortiori, we obtain from here the extimate $(*):\|R(\lambda, A)\| \leq c_{\alpha} /|\lambda|$, for all $\lambda$ in any triangular sector $\sum_{\alpha}$ of $\mathbf{C}^{+}$ defined by $\sum_{\alpha}=\left\{\lambda \in \mathbf{C}^{+}:|\arg \lambda| \leq \alpha\right\}$ for any angle $0<\alpha<\frac{\pi}{2}$, where $c_{\alpha}=1 / \cos \alpha \nearrow+\infty$, as $\alpha \nearrow \frac{\pi}{2}$. (ii) Next, estimate (2.32) on the imaginary axis produces the estimate $\left({ }^{* *}\right):\|R(\lambda, A)\| \leq c_{\beta} /|\lambda|$ for all $\lambda$ in the cone $K_{\beta}=\left\{\lambda \in \mathbf{C}: \frac{\pi}{2}-\beta<|\arg \lambda|<\frac{\pi}{2}+\beta\right\}$, for some angle $0<\beta<\frac{\pi}{2}$, by classical Taylor expansion on the (analytic) resolvent operator $R(\cdot, A)$ centered at $\lambda=i \omega$. Combining estimate $(* *)$ with estimate $\left(^{*}\right)$ for a suitable $\alpha$ with $\frac{\pi}{2}>\alpha>\frac{\pi}{2}-\beta$ yields the classical resolvent characterization for analyticity as in [F.1, p. 188], [P.1, p. 61] on $\sum_{\alpha} \cup K_{\beta}$.

In turn, estimate (2.32) is established, one we show that: given $\epsilon>0$, there exists $C_{\epsilon}>0$ such that, for all $\omega \in \mathbf{R},|\omega| \geq$ some $\omega_{0}>0$, the vector $y(\omega)$ in (2.31) satisfies the following inequality:

$$
\|u(\omega)\|_{H^{2}(\Omega)}^{2}+\|v(\omega)\|_{L_{2}(\Omega)}^{2}+\|\theta(\omega)\|_{L_{2}(\Omega)}^{2} \leq \epsilon\|y(\omega)\|_{Y}^{2}+C_{\epsilon}\left\|\frac{f_{0}}{\omega}\right\|_{Y}^{2} .
$$

To achieve (2.33), the basic "driving term" is the estimate (dropping the dependence on $\omega$ )

$$
\frac{1}{|\omega|}\|\theta\|_{H^{1}(\Omega)}^{2} \leq \frac{\epsilon}{2}\|y\|_{Y}^{2}+\frac{1}{2 \epsilon}\left\|\frac{f_{0}}{\omega}\right\|_{Y}^{2}
$$

ESAIM: Proc., Vol. 4, 1998, 199-222 
which readily follows from the structure of $A$. (If one removes from $A$, as a $3 \times 3$ operator matrix, the bottom-right term, corresponding to $\Delta \theta$, one obtains a skew-adjoint operator). Writing $(i \omega I-A)[u, v, \theta]=\left[u_{0}, v_{0}, \theta_{0}\right]=f_{0} \in Y$, explicitly, one obtains the following starting point of three equations.

For B.C. Set \#4:

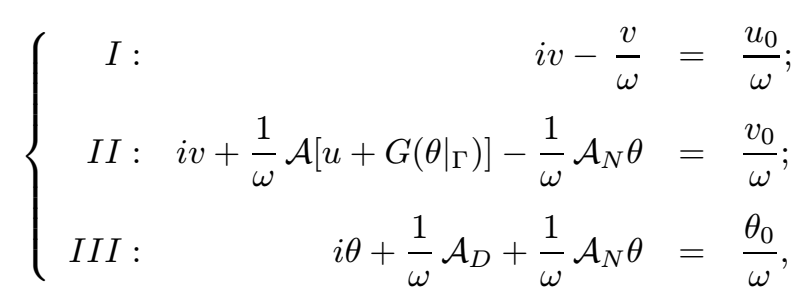

where

$$
\begin{gathered}
\mathcal{A} h=\Delta^{2} h, \mathcal{D}(\mathcal{A})=\left\{h \in H^{4}(\Omega) \cap H_{0}^{1}(\Omega): \Delta h+B_{1} h=0 \text { on } \Gamma\right\} ; \\
h=G g \Leftrightarrow\left\{\Delta^{2} h=0 \text { in } \Omega ;\left.h\right|_{\Gamma}=0,\left[\Delta+B_{1} h\right]_{\Gamma}=g\right\} . \\
\mathcal{A}_{D} h=-\Delta h, \mathcal{D}\left(\mathcal{A}_{D}\right)=H^{2}(\Omega) \cap H_{0}^{1}(\Omega) ; \\
\mathcal{A}_{N} h=-\Delta h, \mathcal{D}\left(\mathcal{A}_{N}\right)=\left\{h \in H^{2}(\Omega):\left[\frac{\partial h}{\partial \nu}+b h\right]_{\Gamma}=0\right\} .
\end{gathered}
$$

\section{For B.C. Set \#5:}

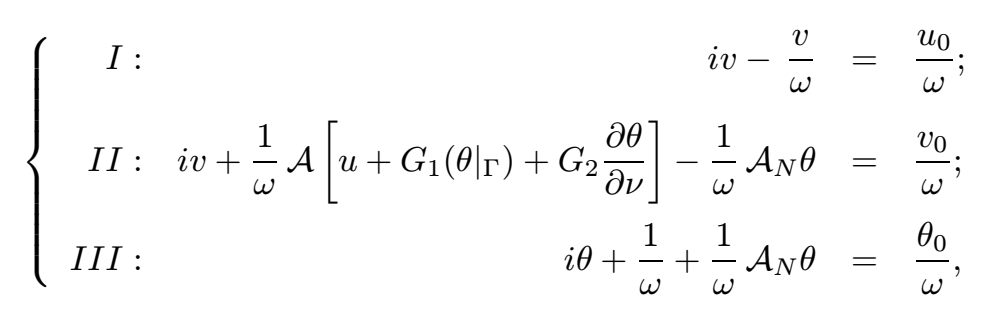

where now

$$
\begin{gathered}
\mathcal{A} h=\Delta^{2} h, \mathcal{D}(\mathcal{A})=\left\{h \in H^{4}(\Omega):\left[\Delta h+B_{1} h\right]_{\Gamma}=0 ;\left[\frac{\partial \Delta h}{\partial \nu}+B_{2} h-h\right]_{\Gamma}=0\right\} ; \\
h=G_{i} g \Longleftrightarrow\left\{\Delta^{2} h=0 ; \Delta h+B_{1} h=\left\{\begin{array}{ll}
g & \text { if } i=1 \\
0 & \text { if } i=2
\end{array} ;\right.\right. \\
{\left[\frac{\partial \Delta h}{\partial \nu}+B_{2} h-h\right]=\left\{\begin{array}{ll}
0 & \text { if } i=1 \\
g & \text { if } i=2
\end{array}\right\} .}
\end{gathered}
$$


The general idea to prove estimate (2.33) is as follows: we seek to combine the 'driving' $\epsilon$-estimate (2.34) with a-priori bounds for $u, v, \theta$ in the right norms, in order to dominate each norm quantity $\|q\|$ of interest, as follows

$$
\|q\| \leq[a+b]\left[\epsilon a+k_{\epsilon} b\right] \leq 2 \epsilon a^{2}+C_{\epsilon} b^{2}, a, b \geq 0
$$

to be specialized with $a=\|y\|_{Y}$ and $b=\left\|\frac{f_{0}}{\omega}\right\|_{Y}$.

Step 1. The first part of the proof is in common for B.C. Set \#4 and \#5. It yields a-priori bounds for $v, \theta$, and $u$ (in this order), $|\omega| \geq 1$ :

$$
\left\{\begin{aligned}
\frac{1}{|\omega|}\|v\|_{H^{2}(\Omega)}+\frac{1}{\sqrt{|\omega|}}\|v\|_{H^{1}(\Omega)} & \leq C\left[\|y\|_{Y}+\left\|\frac{f_{0}}{\omega}\right\|_{Y}\right] ; \\
\frac{1}{|\omega|}\|\theta\|_{H^{2}(\Omega)} & \leq C\left[\|y\|_{Y}+\left\|\frac{f_{0}}{\omega}\right\|_{Y}\right] ; \\
\frac{1}{|\omega|}\|u\|_{H^{4}(\Omega)}+\frac{1}{\sqrt{|\omega|}}\|u\|_{H^{3}(\Omega)} & \leq C\left[\|y\|_{Y}+\left\|\frac{f_{0}}{\omega}\right\|_{Y}\right] .
\end{aligned}\right.
$$

Inequality (2.50) is obtained through P.D.E. estimates for both problems \#4 or \#5.

More precisely, the key is to show that

$$
\left\|\Delta^{2}\left(\frac{u}{\omega}\right)\right\|_{L_{2}(\Omega)}+\left\|\Delta\left(\frac{u}{\omega}\right)\right\|_{H^{\frac{3}{2}(\Gamma)}} \leq C\left[\|y\|_{Y}+\left\|\frac{f_{0}}{\omega}\right\|_{Y}\right] .
$$

Step 2. The following fundamental estimate holds true for B.C. Set \#4: Given $\epsilon>0$, there is $C_{\epsilon}>0$, such that $\forall \omega \in \mathbf{R}$ with $|\omega| \geq 1$ :

$$
\left|\frac{1}{\omega}\left(\mathcal{A}_{D} v, \theta\right)_{L_{2}(\Omega)}\right| \leq \epsilon\|y\|_{Y}^{2}+C_{\epsilon}\left\|\frac{f_{0}}{\omega}\right\|_{Y}^{2} .
$$

Instead, for B.C. Set \#5, one can only write

$$
\left|\frac{1}{\omega}(\Delta v, \theta)_{L_{2}(\Omega)}\right| \leq \epsilon\|y\|_{Y}^{2}+C_{\epsilon}\left\|\frac{f_{0}}{\omega}\right\|_{Y}^{2} .
$$

These bounds are obtained by combining the 'driving' $\epsilon$-estimate (2.34) with the $a$-priori bounds in Step 1.

Step 3. Via Eqn. III in each case and Step 2, one then obtains the desired estimate (2.33) for $\theta$. Moreover, one improves as a corollary upon prior a-priori bounds to obtain:

(i) for both cases, B.C. \#4 and 5:

$$
\left|\frac{1}{\omega}\left(\mathcal{A}_{N} \theta, v\right)_{L_{2}(\Omega)}\right| \leq \epsilon\|y\|_{Y}^{2}+C_{\epsilon}\left\|\frac{f_{0}}{\omega}\right\|_{Y}^{2}
$$

ESAIM: Proc., Vol. 4, 1998, 199-222 
(ii) For B.C. Set \#4, where $v \in \mathcal{D}\left(\mathcal{A}_{D}\right)=H^{2}(\Omega) \cap H_{0}^{1}(\Omega)$ :

$$
\frac{1}{|\omega|}\|v\|_{H^{1}(\Omega)}^{2} \leq \epsilon\|y\|_{Y}^{2}+C_{\epsilon}\left\|\frac{f_{0}}{\omega}\right\|_{Y}^{2} .
$$

For B.C. Set \#5, where $v \in \mathcal{D}\left(\mathcal{A}^{\frac{1}{2}}\right)=H^{2}(\Omega)$ :

$$
\left|\frac{1}{\omega}(\Delta v, v)_{L_{2}(\Omega)}\right| \leq \epsilon\|y\|_{Y}^{2}+C_{\epsilon}\left\|\frac{f_{0}}{\omega}\right\|_{Y}^{2},
$$

where $(2.54 \mathrm{~b})$ is a weaker result than $(2.54 \mathrm{a})$.

Step 4. From here on, the proof for B.C. \#4 and the proof for B.C. \#5 bifurcate and go their separate ways, since in the former case the variable $v$ is 'good' because of the $\epsilon$-estimate (2.54a); while in the latter case, the variable $v$ is not good enough, because of the weaker estimate $(2.54 \mathrm{~b})$. As a sign of departure: the next step is to take the $L_{2}(\Omega)$-inner product of Eqn. II with the 'good' variable $v$ in the case of B.C. \#4, leading after further P.D.E. analysis to the desired conclusions (2.33); instead, in the case of B.C. \#5, we must carry out the argument still with the good variable $\theta$ (see the 'driving' $\epsilon$-estimate (2.34)), and, accordingly, take the inner product of Eqn. II with $\theta$ instead. In the case of B.C. \#5, one obtains next, after further P.D.E. analysis, the estimate

$$
\left|\|v\|_{L_{2}(\Omega)}^{2}-\left\|\mathcal{A}^{\frac{1}{2}} u\right\|_{L_{2}(\Omega)}^{2}\right| \leq \epsilon\|y\|_{Y}^{2}+C_{\epsilon}\left\|\frac{f_{0}}{\omega}\right\|_{Y}^{2},
$$

still without making use - up to this stage - of the structure of the boundary operators.

Step \#5 (B.C. \#5) To improve (2.55) and obtain (simultaneously) the desired estimate (2.33) for $u$ and $v$, it is critical to take advantage of the structure of the boundary operator $B_{1}$ in $(1.7 \mathrm{~d})$ and rewrite it as

$$
\text { on } \Sigma: B_{1} w=(1-\mu)\left[D_{\tau}^{2} w+c(x) \frac{\partial w}{\partial \nu}\right],
$$

where $D_{\tau}^{2}$ denotes the second tangential derivative. The key final step consists in showing that

$$
\|\Delta u\|_{L_{2}(\Omega)}^{2}+\left\|\left.u\right|_{\Gamma}\right\|_{H^{\frac{3}{2}}(\Gamma)}^{2} \leq \epsilon\|y\|_{Y}^{2}+C_{\epsilon}\left\|\frac{f_{0}}{\omega}\right\|_{Y}^{2},
$$

after which one appeals to elliptic theory to obtain (2.33) for $u$ and - via (2.55) (2.33) for $v$ as well. It is at the level of showing (2.57) for $\left.u\right|_{\Gamma}$ that (2.56) is used. Details are in [L-T.2]. 


\section{Case $\gamma>0$ : Group-like structural property (in the mechanical part) of the s.c. semigroup}

If the mechanical equation (1.1) accounts for rotational forces, $\gamma>0$, and thus becomes the hyperbolic Kirchoff equation rather than the Euler-Bernoulli equation, then the corresponding s.c. semigroup of Theorem 1.1 is not analytic any longer (as pointed out in [T.1] for hinged B.C.); and, in fact, it has a group-like structural property for the mechanical part; in particular, it is neither compact, nor differentiable nor uniformly continuous for all $t>0[\mathrm{Cg}-\mathrm{T}]$, [LT.3]. Indeed, in the canonical case of hinged B.C., there exists an infinite dimensional invariant subspace where the s.c. semigroup restricts to a group. The latter analysis expands on the results obtained in [H.1] in the case of a onedimensional thermo-elastic rod, where the eigenvalues approach asymptotically a vertical line. Reference [L-T.3] provides a general result stating qualitatively as follows: if $\gamma>0$, then the corresponding thermo-elastic s.c. semigroup (of Theorem 1.1) is the sum of a component, which is a s.c. group in the mechanical part, plus a (possibly, fractional) differentiable, hence compact perturbation, under all Sets \#2 through \#5 of B.C.

\section{Thermal control in the Neumann/Robin B.C. and boundary observation: Control operator $B$ and observation operator $R$ both genuinely unbounded}

We conclude by presenting an optimal control problem with boundary control and boundary observation, where the property that the thermo-elastic semigroup (with $\gamma=0$ in (1.1)) is analytic (see Theorem 1.2) is critical to obtain a full theory, including the corresponding Algebraic Riccati Equation, by falling into an established abstract theory [B-D-D-M], [D-I.1], [L-T.4], [L-T.5]. For sake of definiteness, we shall consider B.C. Set \#3, with thermal control in the Neumann/Robin B.C.

$$
\begin{cases}w_{t t}+\Delta^{2} w+\Delta \theta=0 & \text { in }(0, T] \times \Omega \equiv Q ; \\ \theta_{t}-\eta \Delta \theta-\Delta w_{t}=0 & \text { in } Q ; \\ w(0, \cdot)=w_{0} ; w_{t}(0, \cdot)=w_{1} ; \theta(0, \cdot)=\theta_{0} & \text { in } \Omega ; \\ w \equiv \frac{\partial w}{\partial \nu} \equiv 0 & \text { on }(0, T] \times \Gamma \equiv \Sigma ; \\ \frac{\partial \theta}{\partial \nu}+b \theta=u & \text { on } \Sigma, b \geq 0 .\end{cases}
$$

ESAIM: Proc., Vol. 4, 1998, 199-222 
We explicitly take below the Robin case with constant $b>0$. The present problem is affected by a thermal boundary control $u \in L_{2}\left(0, T ; L_{2}(\Gamma)\right) \equiv L_{2}(\Sigma)$ acting on a suitable linear combination of the temperature $\theta$ and its flux $\frac{\partial \theta}{\partial \nu}$. Consistently with the regularity theory, we select the following cost functional to be minimized:

$$
\begin{aligned}
J(u, w, \theta)= & \int_{0}^{T}\left\{\left\|\left.\Delta w(t)\right|_{\Gamma}\right\|_{L_{2}(\Gamma)}^{2}+\left\|\left.w_{t}(t)\right|_{\Gamma}\right\|_{L_{2}(\Gamma)}^{2}+\left\|\left.\theta(t)\right|_{\Gamma}\right\|_{L_{2}(\Gamma)}^{2}+\right. \\
& \left.\|u(t)\|_{L_{2}(\Gamma)}^{2}\right\} d t+a\left\|\left\{w(T), w_{t}(T), \theta(T)\right\}\right\|_{H_{0}^{2}(\Omega) \times L_{2}(\Omega) \times L_{2}(\Omega)}^{2},
\end{aligned}
$$

where $\left\{w_{0}, w_{1}, \theta_{0}\right\} \in H_{0}^{2}(\Omega) \times L_{2}(\Omega) \times L_{2}(\Omega)$; moreover, $a=0$ if $T=\infty$ and $a=1$, if $T<\infty$. For $T=\infty$, problem (4.1), (4.2) is a purely boundary problem, with boundary control and boundary observation. Problem (4.1) can be rewritten abstractly as $\dot{y}=A y+B u$, with observation operator $R$ to be the following (Dirichlet) trace operator:

$$
R f=\left\{\left.\Delta f_{1}\right|_{\Gamma},\left.f_{2}\right|_{\Gamma},\left.f_{3}\right|_{\Gamma}\right\}, f=\left\{f_{1}, f_{2}, f_{3}\right\} .
$$

$R$ : continuous $H_{0}^{\frac{5}{2}+\epsilon}(\Omega) \times H_{0}^{\frac{1}{2}+\epsilon}(\Omega) \times H^{\frac{1}{2}+\epsilon}(\Omega) \rightarrow Z=L_{2}(\Gamma) \times L_{2}(\Gamma) \times L_{2}(\Gamma)$,

with $\epsilon>0$ arbitrary, and finial state observation $G=I$. An analysis, carried out in [L-T.5, Chapter 3, Section 11.2] shows that

$$
\begin{gathered}
A^{-\rho} B \in \mathcal{L}(U ; Y), \text { hence } A^{-\rho} B \in \mathcal{L}(U ; Y) ; \rho=\frac{1}{4}+\epsilon, \\
R: \text { continuous } \mathcal{D}\left((-A)^{\delta}\right) \rightarrow Z, \delta=\frac{1}{4}+\frac{\epsilon}{2}, \\
\mathcal{D}\left((-A)^{\delta}\right)=H_{0}^{\frac{5}{2}+\epsilon}(\Omega) \times H_{0}^{\frac{1}{2}+\epsilon}(\Omega) \times H^{\frac{1}{2}+\epsilon}(\Omega), \delta=\frac{1}{4}+\frac{\epsilon}{2},
\end{gathered}
$$

where, since $\rho=\frac{1}{4}+\epsilon$ by (4.5), we have

$$
\frac{1}{4}+\frac{\epsilon}{2}=\delta<\left\{\frac{1}{2}, 1-\rho\right\}, 1-\rho=\frac{3}{4}+\epsilon
$$

as required by the available abstract theory [B-D-D-M], [L-T.5, Chapters 1 and 2]. Thus, such abstract treatment applies and yields a full Riccati equation/regularity theory for the optimal control problem (4.1), (4.2), see [L-T.5, Chapters 1 and 2]. One may also consider a min-max game theory problem with boundary control/boundary disturbance and boundary observation for Eqn. (4.1), to which the abstract theory of [M-T] and [L-T.5, Chapter 6] applies.

\section{References}

[A.1] G. Avalos, Exact/approximate controllability for a thermo-elastic plate, with thermal control, preprint November 1997. 
[A-L.1] G. Avalos and I. Lasiecka, Exponential stability of a thermo-elastic system without mechanical dissipation, IMA Preprint Series \#1357, November 1995, University of Minnesota, Randiconti dell'Istituto di Matematica dell' Universitā di Trieste, special issue in memoriam of Pierre Grisvard, suppl. Vol. XX VII (1997), 1-28.

[A-L.2] G. Avalos and I. Lasiecka, Exponential stability of a thermo-elastic system without mechanical dissipation II: The case of simply supported boundary conditions, IMA Preprint \#1397, March 1996, University of Minesota, SIAM J. Math. Anal., Vol 29, 1998, 155182 .

[A-L.3] G. Avalos and I. Lasiecka, Uniform decay rates in nonlinear thermoelastic systems without mechanical dissipation, in Proceedings IFIP Conference on Optimal Control: Theory, Algorithms, and Applications, Gainesville, FL. Kluwer Academic Publishers, Norwell, MA, February 1997.

[B-D-D-M] A. Bensoussan, M. Delfour, G. Da Prato, S. Mitter, Representation and Control of Infinite Dimensional Systems, Birkhäuser, 1993.

[B-L-Z] J. A. Burns, Z. Liu, and S. Zheng, On the energy decay of a linear thermoelastic bar, J. Math. Anal. Appl. 179(2) (1993), 574-591.

[Cg-T.1] S. K. Chang and R. Triggiani, Spectral analysis of thermo-elastic plates with rotational forces, presented at Proceedings IFIP Conference on Optimal Control: Theory, Algorithms, and Applications, Gainesville, FL. Kluwer Academic Publishers, Norwell, MA, February 1997.

[C-T.1] S. Chen and R. Triggiani, Proof of two conjectures of G. Chen and D. L. Russell on structural damping for elastic systems. The case $\alpha=\frac{1}{2}$. Springer-Verlag Lecture Notes in Mathematics \#1354, 1988, Proceeding of "Seminar in Approximation and Optimization" held at the University of Havana, Cuba, January 12-14, 1987.

[C-T.2] S. Chen and R. Triggiani, Proof of extensions of two conjectures on structural damping for elastic systems. The case $\alpha \geq \frac{1}{2}$. Pacific $J$. of Mathematics 136 (1989), 15-55.

[D.1] C. Dafermos, On the existence and the asymptotic stability of solutions to the equations of linear thermoelasticity, Arch. Rational Mech. Anal. 29 (1968), 241-271.

[D-I.1] G. Da Prato and Ichikawa, Riccati equations with unbounded coefficients, Ann. Math. Pura Appl. 140 (1985), 209-221.

[F.1] H. O. Fattorini, The Cauchy Problem, Encyclopedia of Mathematics and its Applications, Addison-Wesley Publishing Company, 1983.

ESAIM: Proc., Vol. 4, 1998, 199-222 
[H.1] S. W. Hansen, Exponential energy decay in a linear thermoelastic rod, J. Math. Anal. Appl. 167 (1992), 429-442.

[H-L-P.1] D. Henry, O. Lopes, and A. Perrissinotto, On the essential spectrum of a semigroup of thermoelasticity, Nonlinear Anal. T. M. A. (1993), $65-75$.

[H-Z.1] S. W. Hansen and Zhang, Boundary control of a linear thermoelastic rod, J. Math. Anal. Appl. 210 (1997), 182-205.

[K.1] J. U. Kim, On the energy decay of a linear thermo-elastic bar and plate, SIAM J. Math. Anal. 23 (1992), 889-899.

[K.2] S. G. Krein, Linear Differential Equations in Banach Space, Trans. Amer. Math. Soc., vol. 29, Amer. Math. Society, Providence, RI 02904, 1971.

[Lag.1] J. Lagnese, Boundary Stabilization of Thin Plates, SIAM, Philadelphia (1989).

[Lag.2] J. Lagnese, The reachability problem of thermo-elastic plates, Arch. Rat. Mech. Analysis 112(1990), 223-267.

[Lag-Lio] J. Lagnese and J. L. Lions, Modeling, Analysis, and Control of Thin Plates, Masson, Paris, 1988.

[Las] I. Lasiecka, Control and stabilization of interactive structures, Systems and Control in the 21st Century, Birkhauser (1997), 245-262.

[L-T.1] I. Lasiecka and R. Triggiani, Two direct proofs of analyticity of the s.c. semigroup arising in abstract thermo-elastic equations, Advances in Diff. Eqns., to appear. Presented at IFIP Conference, University of Florida, Gainesville, February 1997.

[L-T.2] I. Lasiecka and R. Triggiani, Direct P.D.E. proofs of analyticity of the thermo-elastic semigroups with coupled B.C., submitted. Presented at Workshop on Deterministic and Stochastic Evolutionary Systems, Scuola Normale Superiore, Pisa, Italy, July 1997; and at IFIP Conference, Detroit, July 1997, to appear in Abstract and Applied Analysis.

[L-T.3] I. Lasiecka and R. Triggiani, Lack of compactness and differentiability of the s.c. semigroup arising in thermo-elastic plate theory with rotational forces, submitted. Presented at IFIP Conference, University of Florida, Gainesville, February 1997, to appear in Semigroup Forum.

[L-T.4] I. Lasiecka and R. Triggiani, Differential and Algebraic Riccati Equations with Applications to Boundary/Point Control Problems: Continuous Theory and Approximation Theory, Springer-Verlag Lecture Notes, LNCIS, vol. 164 (1991), 160 pp. 
[L-T.5] I. Lasiecka and R. Triggiani, Control Theory for Partial Differential Equations: Continuous and Approximation Theories, vol. 1, Cambridge University Press, Encyclopedia of Mathematics and its Applications, to appear in 1998.

[L-T.6] I. Lasiecka and R. Triggiani, Exact null controllability of structurally damped and thermo-elastic parabolic models, Memoria, Accademia dei Lincei, Rome (Italy), Sezione Matematica, to appear.

[Leu.1] G. Leugering, A decomposition method for integro-partial equations and applications, J. Math. Pures Appl. 71 (1992), 561-587.

[L-M] J. L. Lions and E. Magenes, Nonhomogeneous Boundary Value Problems and Applications, Springer, Heidelberg, 1972.

[L-L.1] K. Liu and Z. Liu, Exponential stability and analyticity of abstract linear thermo-elastic systems, preprint, December 1996.

[L-R] Z. Liu and M. Renardy, A note on the equations of a thermoelastic plate, Appl. Math. Letters 8 (1995), 1-6.

[Le-Zu.1] G. Lebeau and E. Zuazua, Sur la d'ecroissance non uniforme de l'energie dans le systeme de la thermo elasticite lineaire, C. R. Acad. Sci. Paris, 324(1997), 409-415.

[L-Z.1] Z. Liu and S. Zheng, Exponential stability of semigroup associated with thermoelastic system, Quarterly Appl. Math. LI(3) (1993), $535-545$.

[L-Z.2] Z. Liu and S. Zheng, Exponential stability of the Kirchoff plate with thermal or viscoelastic damping, Quarterly Appl. Math., LV (1997), $551-564$.

[M-T.1] C. McMillan and R. Triggiani, Min-max game theory and Algebraic Riccati Equations for boundary control problems with analtyic semigroups II: General case, Nonlinear Analysis, Theory, Methods, Applications 22 (1994), 431-465.

[P.1] A. Pazy, Semigroups of Linear Operators and Applications to Partial Differential Equations, Springer-Verlag, 1983.

[PM-Z.1] G. Perla Menzala and E. Zuazua, Explicit exponential decay rates for solutions of von Kármán's system of thermoelastic plates, $C . R$. Acad. Sci. Paris 324(1997), 49-54.

[R.1] D. Russell, A general framework for the study of indirect damping mechanisms in elastic systems, J. Math. Anal. Appl. 173(2) (1993), $339-358$.

[S.1] Y. Shibata, On the exponential decay of the energy of a linear thermo-elastic plate, Comp. Appl. Math 13 (1994), 81-102. 
[Sl.1] M. Slemrod, Global existence, uniqueness, and asymptotic stability of classical smooth solutions in one-dimensional non-linear thermoelasticity, Arch. Rat. Mech. Anal. 76 (1981), 97-134.

[T.1] R. Triggiani, Analyticity, and lack thereof, of semigroups arising from thermo-elastic plates, Proceedings of Computational Science for the 21st Century, John Wiley, May 5-7, 1997.

[T.2] R. Triggiani, Sharp regularity of thermo-elastic equations with rotational forces, and boundary control, June 1997, submitted.

[T-Z.1] L. de Teresa and E. Zuazua, Controllability for the linear system of thermoelastic plates, Advances in Diff. Eqns. 1(3) (1996), 369-402.

[Z.1] E. Zuazua, Controllability of the linear system of thermoelasticity, J. Mathematiques Pures et Appl. 74(1995), 303-346. 